\title{
Biotransformation of Rutin to Quercetin by Microorganisms
}

\author{
Zhitang Lu*, Yating Zhang, Nan Shi \\ 1. College of Life Sciences, Hebei University and Key Laboratory of Microbial Diversity Research and \\ Application of Hebei Province, Baoding, China 071002
}

\begin{abstract}
Quercetin is a kind of flavonoid drugs, which is produced mainly by direct extracting from Flos Sophorae or acid hydrolysing from rutin. In this study, microorganism strains that can transfer rutin to quercetin were isolated from soil, screened by TLC and re-screened by HPLC. As a result, strain D97 with the highest transformation ability was obtained. The hydrolyze enzyme was intracellular enzyme and transformation rate was $\mathbf{7 5 . 6 \%}$ within a system comprising of 50mg wet cells in $4 \mathrm{~mL}$ of $0.3 \%$ rutin solution and transformed for $72 \mathrm{hr}$ at $25^{\circ} \mathrm{C}$. Strain D97 was identified as Pseudomonas sp. by integrating the $16 \mathrm{~S}$ rDNA phylogeny analysis result and a variety of morphological, physiological and biochemical features.
\end{abstract}

Key words: Rutin; Quercetin; Transformation; Identification

\section{INTRODUCTION}

Flos Sophorae is the dried flowers and buds of Chinese scholartree Sophora japonica L., which originates in the north of China, and widely distribute in the south and southwest areas nowadays. Traditional Chinese medicine considers that Flos Sophorae has a number of drug effects, such as, cooling blood, hemostasis, clearing away heat and reducing fire and so on. The most important use of it is treating hematochezia, metrorrhagia and metrostaxis and liverfire. Recently researches indicate that Flos Sophorae contains abundant physiological active components and nutrients, and shows remarkable curative effect as natural medicine and high quality feed in clinic. Moreover the water extract of Flos Sophorae has significant effect on anti-bacteria, anti-inflammatory, antiviral, etc (Sun et al., 2009).

The major effective components of Flos sophorae are flavonoids composed of large quantity of rutin $(24.46 \%, w / w)$ and little amount of quercetin $(1.41 \%, w / w)$ (Cheng et al., 2004). Both rutin and quercetin are medicines and have many physiological activities. They have similar activities in eliminating free-radicals, antioxidative activities and protective effect in the hypoxia/hypoglycemia model of bacteria precipitation, antioxidative activities in vitro and anti-lipid peroxidation, but quercitin act preponderant function than rutin (Jin et al. 2007, Jin et al. 2009, Su et al. 2002, and Jiang et al. 2007). At present, only little part of Flos sophoraes are used for rutin extraction, most of them are wasted. Rutin is often hydrolyzed by acid to obtain the more active quercetin in modern industry production, and large quantity of acidic waste water is produced. Hence, it is necessary to develop a simple, economical, environment friendly and efficient method for quercetin production.

This study aimed to screen potential bacteria that could transform rutin to quercetin from soil samples collected from a decomposed leaves accumulation area of Chinese scholartree woods. If this procedure comes to reality in industry, it will not only solve the waste of Flos sophorae as resources and improves its additional value, but also can reduce the environmental pollution caused by the quercetin acid hydrolysis technology. 


\section{Zhitang Lu, Yating Zhang, Nan Shi / IOSR Journal of Engineering (IOSRJEN) \\ www.iosrjen.org

Vol. 2 Issue 1, Jan.2012, pp.070-076

\section{MATERIALS AND METHODS}

\subsection{Samples}

Soil samples were collected from a Chinese scholartree woods in Baoding, China.

\subsection{Medium and reagent}

Nutrient agar medium (pH7.0) was used for bacteria isolation and cultivation. Rutin (AR), standard reagents of rutin and quercetin were purchased from Chengdu Must Bio-Technology Co., Ltd.

\subsection{Isolation of quercetin-producing bacteria}

$10 \mathrm{~g}$ soil sample was suspended in sterile water ( $\mathrm{pH} 7.0$ ) with 20 glass beades and oscillated at $25^{\circ} \mathrm{C}$ for 25 min. Quercetin-producing bacteria were isolated using serial dilution method by spread suitable dilutions on Nutrient agar medium supplemented with $0.1 \%(w / w)$ rutin. After incubate for $1 \mathrm{~d}$ at $25^{\circ} \mathrm{C}$, bacterium-like colonies were inoculated, and those colonies with deep-colored hydrolysis zones after sprayed $1.0 \% \mathrm{AlCl}_{3}$-ethanol solution were selected for screening.

\subsection{Screening techniques}

\subsubsection{Preliminary screening}

Thin layer chromatography (TLC) method was used to screen the active strains. The isolate was cultured in $4 \mathrm{~mL}$ of Nutrient broth ( $\mathrm{pH} 7.0)$ in $15 \times 150 \mathrm{~mm}$ test tubes. Cultivation was conducted at $25^{\circ} \mathrm{C}$ in a thermostat shaker by shaking at $280 \mathrm{r} / \mathrm{min}$ for $1 \mathrm{~d}$. The liquid culture was centrifuged at $4500 \mathrm{r} / \mathrm{min}$ for $5 \mathrm{~min}$ at room temperature $\left(24^{\circ} \mathrm{C} \pm 1^{\circ} \mathrm{C}\right)$ to separate the cell and the supernatant. The cell pellet was washed with $2 \mathrm{~mL}$ of $0.1 \mathrm{~mol} / \mathrm{L}$ phosphate buffer ( $\mathrm{pH} 7.0$ ) and centrifuged again to harvest the biomass. Then the bacteria biomass was suspended with $4 \mathrm{~mL}$ of phosphate buffer (pH7.0). $4 \mathrm{~mL}$ of cell suspension and the fermentation supernatant were supplemented with $0.3 \%$ rutin and react for $3 \mathrm{~d}$ by shaking at $280 \mathrm{r} / \mathrm{min}$ and $25^{\circ} \mathrm{C}$, repetitively. At last, TLC analysis was performed to check the activity of transferring rutin to quercetin.

The TLC analysis was carried out by loaded the sample on polyamide film and developed with 75\% microemulsion consisted of SDS/n-butyl alcohol/n-heptane/water, 0.27: 0.63: 0.10: $36(\mathrm{~m} / \mathrm{m})($ Kang et al., 2000). The developed polyamide film was stained by spraying $1 \% \mathrm{AlCl}_{3}$-ethanol solution and dried under room temperature. TLC polyamide film were scanned and analyzed by UN-scan-it gel scanning software (SIM International Group Co. LTD. U.S.A.).

\subsubsection{Secondary screening}

After preliminary screening, High Performance Liquid Chromatography (HPLC) was used to re-screen the active strains by comparing the retained time of converted products with quercetin standard. The chromatography conditions were as following: separated by pre-packed Hypersil ODS (C18) column ( $250 \times 4.6 \mathrm{~mm}$, Elite, China) column at $24^{\circ} \mathrm{C} \pm 1{ }^{\circ} \mathrm{C}$ and detected by UV detector at $360 \mathrm{~nm}$, the mobile phase composed of acetonitrile: methanol: water: phosphoric acid (100:10:340:0.3, v/v) was used as the eluant at a flow rate of $1.0 \mathrm{~mL} / \mathrm{min}$, sample volume was $20 \mu \mathrm{L}$ (Jia et al., 2008).

The HPLC sample was prepared as following: the transformed solution was centrifuged to get rid of the cell, and then the supernatant was filtrated by $0.22 \mu \mathrm{m}$ membrane and ready for HPLC. 


\section{Zhitang Lu, Yating Zhang, Nan Shi / IOSR Journal of Engineering (IOSRJEN) www.iosrjen.org

Vol. 2 Issue 1, Jan.2012, pp.070-076

\subsection{Transformation rate determination}

Diluted standards solution of rutin and quercetin were prepared by using $4.90 \mathrm{mg}$ rutin and $5.88 \mathrm{mg}$ quercetin and they were used for preparation of different working standards using methanol.

$20 \mu \mathrm{L}$ of the standard solution of each concentration (rutin solution: $0.272 \mu \mathrm{g} / \mathrm{mL}, 0.544 \mu \mathrm{g} / \mathrm{mL}$, $1.089 \mu \mathrm{g} / \mathrm{mL}, 2.178 \mu \mathrm{g} / \mathrm{mL}, 4.356 \mu \mathrm{g} / \mathrm{mL}$; quercetin solution: $0.327 \mu \mathrm{g} / \mathrm{mL}, 0.653 \mu \mathrm{g} / \mathrm{mL}, 1.307 \mu \mathrm{g} / \mathrm{mL}$, $2.613 \mu \mathrm{g} / \mathrm{mL}, 5.227 \mu \mathrm{g} / \mathrm{mL}$ ) were analyzed by HPLC. Standard curves were manufactured with the concentration of standard solutions as abscissa and the average peak area of three times as ordinate.

Transformation of rutin to quercetin was carried out by cell biomass as described in 2.4.1.The HPLC sample was prepared as described in 2.4.2. After injected three times, the average peak area of the sample was obtained from the liquid chromatogram and then the concentration of quercetin was figured out through the standard curve of quercetin and the starting rutin concentration of the transfermation system.

\subsection{Taxonomical Investigations of active strain}

The identification of bioactive strain was conducted based on 16S rDNA phylogenetic analysis and morphological observation, as well as determination of physiological and biochemical features.

The genomic DNA of tested strain was extracted with phenol-chloroform method (Marmur, 1961), 16S rDNA was amplified by polymerase chain reaction using universal forward primer 27F (5'-AGAGTTTGATCMTGGCTCAG-3') and reverse primer 1525R (5'-AGAAAGGAGGTGWT CCARCC-3') (Lane, 1991) with described procedure (Lu et al., 2001). Purified PCR products were directly sequenced by Beijing Sunbiotech Corporation. The sequence obtained was initially estimated by the BLAST facility of NCBI (www.ncbi.nlm.nih.gov/BLAST) and then aligned with all related sequences obtained from GenBank by BioEdit (Hall, 1999). Evolutionary distance matrices were calculated by using the method of Kimura 2-parameter and a neighbour-joining tree was reconstructed by the Mega 5.1 program (Saitou \& Nei, 1987, Tamura et al., 2011).

Cell morphology was examined with a light microscopy (Olympus, BH-2). Presence of spore was examined by staining using Schaeffer-Fulton method, and Gram reaction was determined using the bioMe'rieux Gram Stain kit according to the manufacturer's instructions (Beijing Land Bridge Technology Co., Ltd). All other phenotype determinations were carried out according to Tvrzova' et al.(2006).

\section{RESULTS}

\subsection{Screening result of active strain}

25 bacteria strains and 8 fungi strains were isolated on the Nutrient agar plate at $25^{\circ} \mathrm{C}$, and four of which exhibited obvious quercetin chromatography dots by TLC screening (Fig.1).

The conversion capabilities of the four strains were re-screened by HPLC with rutin and quercetin standards as control. Retained time of rutin was 5.277 min, and retained time of quercetin was 19.944 min (Fig.3). The results suggested that rutin and quercetin could be separated effectively under the experiment condition. Among the four positive strains of preliminary screened, strain D97 had an evident elution peak corresponding to quercetin and showed the highest conversion ability (Fig.2). 


\section{Zhitang Lu, Yating Zhang, Nan Shi / IOSR Journal of Engineering (IOSRJEN) www.iosrjen.org

Vol. 2 Issue 1, Jan.2012, pp.070-076

Thus strain D97was selected for further analysis quantitatively.

After the cultivation of strain D97 in the Nutrient broth for 24h, both the fermentation supernatant and suspension of cell precipitation were prepared and checked conversion activity. The quercetin was only found in cell precipitation transformation system, which suggested that the active enzyme was intracellular enzyme.

Standard curves were manufactured by Excel2003 in the further quantitative study. The regression equation of rutin was $y=12002 x+946.85\left(R^{2}=0.9977\right)$, and the regression equation of quercetin was $\mathrm{y}=26141 \mathrm{x}+1812.8\left(\mathrm{R}^{2}=0.9974\right)$ (Fig.3). The results showed that rutin and quercetin had good linearities within the tested concentration range. The transformation rate was calculated based on the standard curve and the starting concetraion of rutin, as well as the final concetraion of quercetin. A high transformation rate of $75.6 \%$ was reached when transformed by the $50 \mathrm{mg}$ wet cell for $72 \mathrm{hr}$ at $25^{\circ} \mathrm{C}$ in the system of $4 \mathrm{~mL}$ containning $0.3 \%$ rutin.

\subsection{Identification result of strain $D 97$}

Nearly complete $16 \mathrm{~S}$ rDNA sequence of $1491 \mathrm{bp}$ of D97 strain was obtained. When comparing the $16 \mathrm{~S}$ rDNA sequence with the GenBank database, the greatest similarity of $99.2 \%$ was found between strain D97 and Pseudomonas moraviensis (AY970952). In the neighbour-joining phylogenetic tree, strain D97 and Pseudomonas moraviensis CCM $7280^{\mathrm{T}}$ (AY970952) clustered together with a high bootstrap value of $95 \%$ (Fig.4).

Strain D97 is Gram negative and rod bacterium in size of $0.7 \sim 0.9 \times 2.4 \sim 3.0 \mu \mathrm{m}$. The colony is round and wet with irregular edge and in white or translucent color. No spore is formed. Motile by polar flagella. Growth occurs at $10-37^{\circ} \mathrm{C}$, with optimum growth at $28-35^{\circ} \mathrm{C}$. Oxidase is positive, urease and DNase are negative. Tween 80 , gelatin and tyrosine are hydrolysed, but not aesculin or starch. Lecithinase and indole are not produced. Fermentation of D-glucose and assimilation of adipate are positive. Malate is assimilated, but not citrate. Can use glycerol, L-arabinose, D-glucose, D-fructose, D-lyxose as sole carbon sources, but not D-trehalose, D-maltose, D-lactose, D-melibiose and D-sucrose. Tween 80, gelatin and starch are hydrolyzed. Can not reduce nitrate to nitrite. All these phenotype characteristics serve to classify strain D97 as a member of the genus Pseudomonas sp..

\section{DISCUSSION}

TLC method was widely used in separation and determination of flavonoids including rutin and quercetin (Zhou et al., 2006). In this research, we obtained four strains that had the potential activity of transforming rutin to quercetin. But when they were checked by HPLC, only strain D97 exhibited a strong activity. The result indicated that TLC method only fit for preliminary screening of active strains. Although the transformation rate of strain D97 from rutin to quercetin is not as high as the industrial production needed, this wild type bacterium will illustrate a good prospect of application after breeding and optimization of transformation conditions.

The active strain D97 of this research was identified to be Pseudomonas sp., i.e. a bacterium, which is different from other strains that reported mainly fungus and actinomycetes. From another point of view, we found a new microbial resource for biotransformation of rutin to quercetin, and will offer a new technology for quercetin production. However, the enzymology characteristics, conversion conditions and breeding of the higher active strain ect, further studies are still needed to be carried out for this 


\section{Zhitang Lu, Yating Zhang, Nan Shi / IOSR Journal of Engineering (IOSRJEN) www.iosrjen.org

Vol. 2 Issue 1, Jan.2012, pp.070-076

strain.

\section{References}

[1] Cheng Xiuming, Chen Xuelan, Gao Yanhui. et al. (2004). Determined the contents of rutin and quercetin by RP-HPLC. Chinese Traditional Patent Medicine, 26(8), 680-682.

[2] Hall, T. A. (1999). BioEdit, a user-friendly biological sequence alignment editor and analysis program for Windows 95/98/NT. Nucleic Acids Symp Ser, 41, 95-98.

[3] Jia Dongdong, Li Shufen, Yang Honglin. (2008). Determined the contents of rutin and isoquercetin by RP-HPLC. Food Science, 29(8), 499-501.

[4] Jiang, P. Burczynski, F. Campbell, C. et al. (2007). Rutin and flavonoid contents in three buckwheat species Fagopyrum esculentum, F. tataricum, and their protective effects against lipid peroxidation. Food Research International, 40(3), 356-364.

[5] Jin Yue, Lü Yong, Han Guozhu. et al. (2007). Comparative study on in vitro anti-free radical activities of quercetin, isoquercetin, and rutin. Chinese Traditional Patent Medicine, 38(3), 408-412.

[6] Jin Yue, Lü Yong, Han Guozhu. et al. (2009). The study on structure activity relationship and protective effect in the hypoxia/hypoglycemia model of HEK293 cells of quencetin and Glucoside. Chinese Traditional Patent Medicine, 40(4), 618-620.

[7] Kang Chun, Wen Liyu, Ding Zhongbo. (2000). Studies on Separation and Identification of Flavonoid Compositions with Microemulsion Thin Layer Chromatography. Chinese Journal of Pharmaceutical Analysis, 20(2), 121-123.

[8] Kimura, M. (1980). A simple method for estimating evolutionary rates of base substitutions through comparative studies of nucleotide sequences. J Mol Evol, 16, 111-120.

[9]Lane, D. J. (1999). 16S/23S rDNA Sequencing. In: Nucleic acid techniques in bacterial systematics (eds Stackebrandt E, Goodfellow M). John Wiley \& Sons, Chichester, pp115-175.

[10] Lu Zhitang, Liu Zhiheng, Wang Liming, et al. (2001). Saccharopolyspora falva sp. nov. and Saccharopolyspora thermopohila sp.nov., novel actinomycetes from soil. Int J Syst Evol Microbiol, 51, 319-325.

[11] Marmur, J. (1961). A Procedure for the isolation of deoxyribonucleic acid from microorganisms. Journal of Molecular Biology, 3, 208-218.

[12] Saitou, N., Nei M. (1987). The neighbor-joining method: a new method for reconstructing phylogenetic trees. Mol Biol Evol, 4, 406-425.

[13]Su Junfeng, Guo Changjiang. (2002). Comparative study on the antioxidant capacity of quercetin in vivo and in vitro. Chinese Journal of Applied Physiology, 18(4), 382-386.

[14]Sun Guolu, Zhao Qiang, Dong Xiaoning. et al. (2009). Studies on the chemical components and pharmacological activities of Sophora japonica. Journal of Traditional Chinese Veternary Medicine.

[15] Tamura, K., Peterson, D., Peterson, N., et al. (2011). MEGA5: Molecular evolutionary genetics analysis using maximum likelihood, evolutionary distance, and maximum parsimony methods. Mol Biol Evol, 28, 2731-2739.

[16] Tvrzová L, Schumann P, Spröer C, et al. (2006). Pseudomonas moraviensis sp. nov. and Pseudomonas vranovensis sp. nov., soil bacteria isolated on nitroaromatic compounds, and emended description of Pseudomonas asplenii. Int J Syst Evol Microbiol, 56, 2657-2663

[17]Zhou Xuan, Song Fenyun, Zhong Zhaojian. (2006). Determination of Rutin and Quercetin in Flos Sophorae with Thin Layer Chromatogram Scanner. Chinese Journal of Experimental Traditional Medical Formulae, 12(8), 14-16. 
Zhitang Lu, Yating Zhang, Nan Shi / IOSR Journal of Engineering (IOSRJEN) www.iosrjen.org

Vol. 2 Issue 1, Jan.2012, pp.070-076

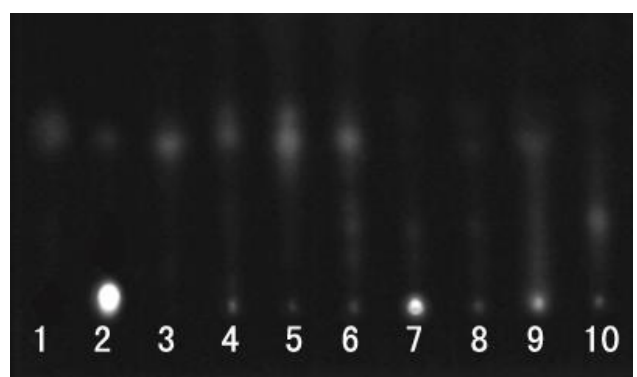

Fig 1. TLC determination result of some bacterial samples

1 rutin, 2 quercetin, 3 negative control, 4-10 samples (7. D97)

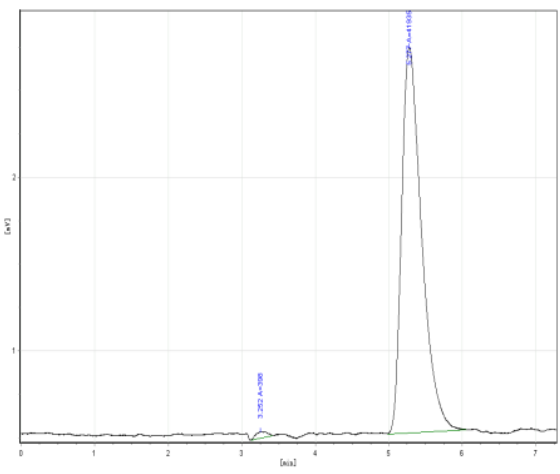

(a)

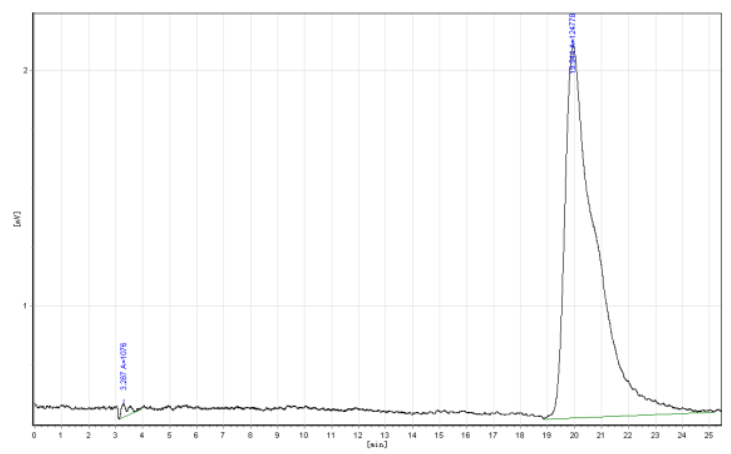

(b)

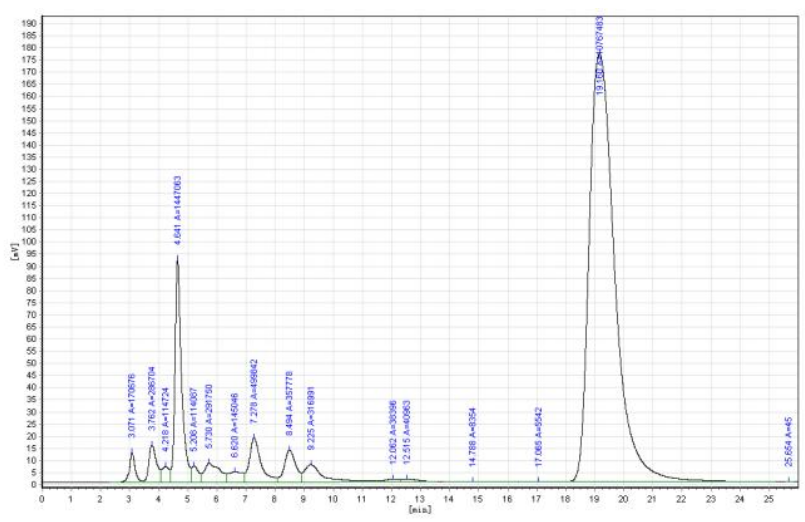

(c)

Fig 2. HPLC chromatograms of rutin standards and tested sample

(a) rutin standard, (b) quercetin standard, (c)tested sample D97
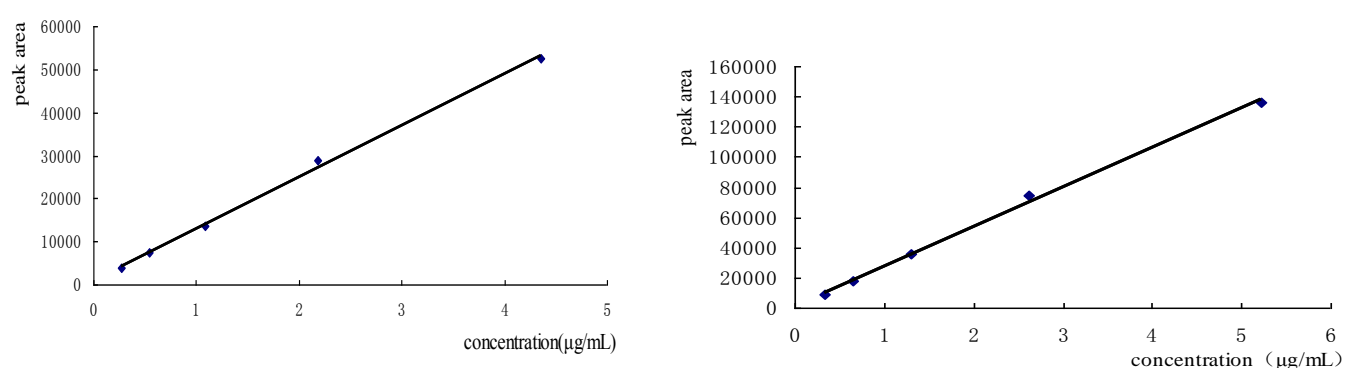

Fig.3 Standard curves of rutin (left) and quercetin (right) by HPLC 
Zhitang Lu, Yating Zhang, Nan Shi / IOSR Journal of Engineering (IOSRJEN) www.iosrjen.org

Vol. 2 Issue 1, Jan.2012, pp.070-076

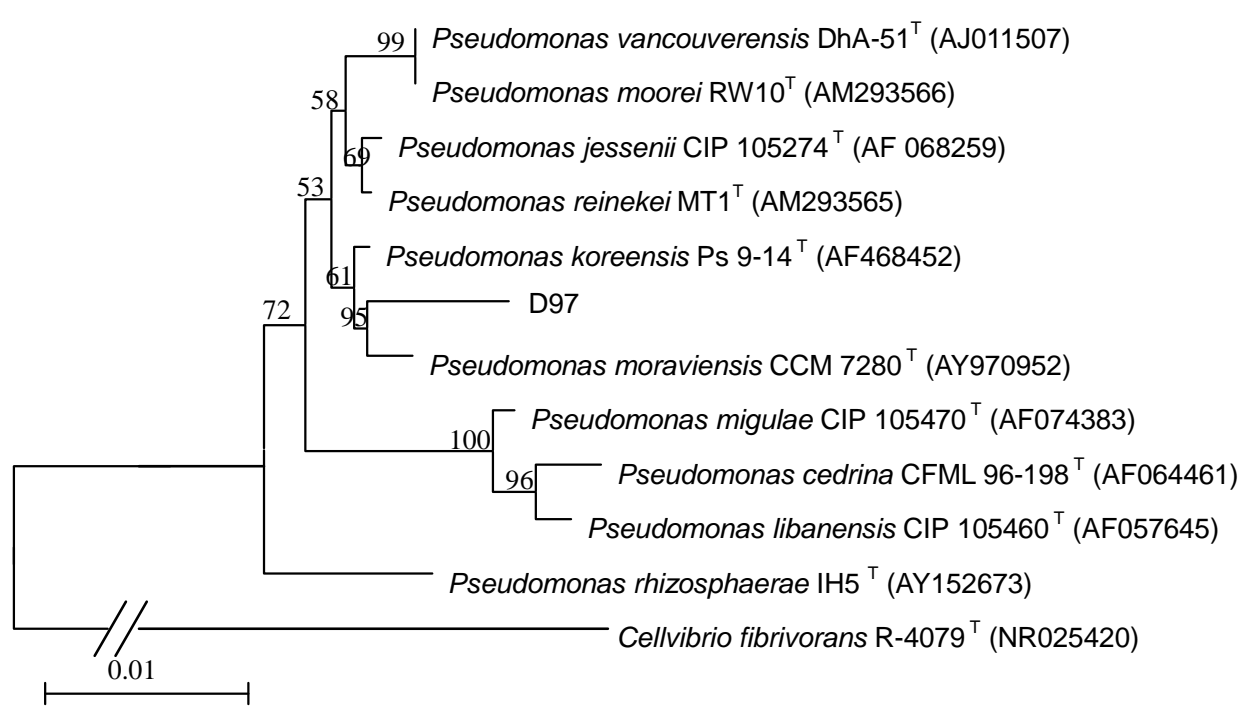

Fig 4. Neighbour-joining phylogenetic tree of strain D97 and related Pseudomonas representatives based on almost complete 16S rDNA sequences using Cellvibrio fibrivorans(NR025420) as outgroup 